\title{
MOTRICIDAD HUMANA: ALGO MÁS QUE UN GIRO LINGÜÍSTICO \\ ¿Qué, del cuerpo y la Educación Física?
}

\section{Alixon David Reyes Rodríguez}

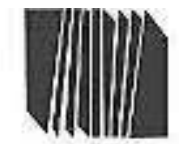

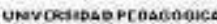
SXYKRIME MI AL LAECR TED of

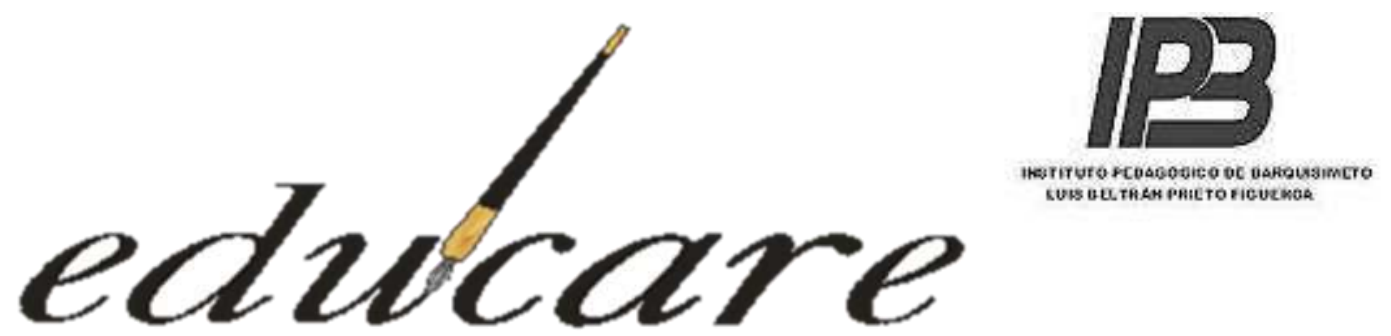

Órgano Divulgativo de la Subdirección de Investigación y Postgrado del Instituto Pedagógico de Barquisimeto "Lıis Beltrám Prieto

Figueroa"

Volumen 23 № 1
MOTRICIDAD HUMANA: ALGO MÁS QUE UN GIRO LINGÜÍSTICO
¿Qué, del cuerpo y la Educación Física?
HUMAN MOBILITY: SOMETHING MORE THAN A LINGUISTIC SHIFT
What, the body and physical education?
I S S $N: 2244-7296$

\section{AUTOR}

Alixon David Reyes Rodríguez Universidad Adventista de Chile 


\section{MOTRICIDAD HUMANA: ALGO MÁS QUE UN GIRO LINGÜÍSTICO ¿Qué, del cuerpo y la Educación Física?}

Alixon David Reyes Rodríguez

pp. $54-84$

\section{$\underline{\text { Revisión Documental }}$}

Recibido:12-12-2018

\section{RESUMEN}

El propósito de este trabajo radica en analizar el estatus del debate que sostiene la representatividad epistémica en el planteamiento que hiciera Manuel Sérgio en referencia a las 'Ciencias de la Motricidad Humana', como propuesta científica enmarcándole en el contexto de un denunciado agotamiento de la Educación Física. Para ello se plantea un trabajo de carácter históricointerpretativo sustentado en la teoría fundamentada, el método dialéctico de Marx y Engels, y la asunción de campo que planteara Bourdieu. Se privilegia la investigación documental con anclajes históricos en el campo de la Educación Física y las Ciencias de la Motricidad Humana, intentando ofrecer algunas aproximaciones en torno a preguntas de investigación: ¿es acaso la motricidad humana, una moda, una tendencia, una corriente?, ¿es una forma de entendernos y situarnos en el mundo?, ¿es una ciencia, tal y como lo plantean Manuel Sérgio y otros investigadores?, ¿cuán sólida se presenta esta propuesta hoy día? Pero, aún más, ¿qué de la Educación Física en este contexto dialéctico?

Palabras clave: motricidad humana, educación física, ciencia, cuerpo, episteme

\section{Alixon David Reyes Rodríguez " Universidad Adventista de Chile}

Aceptado:03-03-2019

\begin{abstract}
The purpose of this work takes root in analyzing the status of the debate that supports the representation epistemica in the exposition that Manuel Sérgio should do in reference to the 'Sciences of the Motricidad Humana ', as scientific offer framing him in the context of a denounced depletion of the Physical Education.For it there appears a work of historical - interpretive character sustained in the based theory, the dialectical method of Marx and Engels, and the field assumption that Bourdieu was raising. The documentary investigation is favoured by historical anchorages in the field of the Physical Education and the Sciences of the Motricidad Humana, trying to offer some approximations concerning questions of investigation: is it perhaps the human motricidad, a mode, a trend, a current?, is it a way of understanding itself and to place in the world?, it is a science, as Manuel Sérgio and other investigators raise it?, how solid does one present this offer today?. But, furthermore, what of the Physical Education in this dialectical context?
\end{abstract}

Keywords: human mobility, physical education, science, body, episteme

*Grupo de Investigación AFSYE, Pedagogía en Educación Física, Universidad Adventista de ChileORCID: 0000-0001-9857-0930 alixonreyes@unach.cl 


\section{MOTRICIDAD HUMANA: ALGO MÁS QUE UN GIRO LINGÜÍSTICO \\ ¿Qué, del cuerpo y la Educación Física?}

Alixon David Reyes Rodríguez

pp. $54-84$

\section{INTRODUCCIÓN}

Este trabajo recoge reflexiones resultantes en el ámbito narrativo histórico e interpretativo en campos como la Educación Física y la motricidad humana, pensando el tema desde las aproximaciones de la arqueología del saber en Foucault (2013, 2002, 1977a), y ello en tanto y cuanto se plantea la búsqueda de rastros de procedencia de la Educación Física y la emergencia de la denominada ciencia de la motricidad humana, rastros que nos permitan reconocer el recorrido histórico y avanzar en la reconstrucción del hilo conductor que otorga cierto rango a aquellos saberes, prácticas, contextos, valores, creencias, representaciones sociales e imaginarios asociados a 'lo corporal', la categoría de disciplina, campo o ciencia (según ciertos autores).

Hay diferencias no menores entre estos tres elementos, a saber: disciplina, campo y ciencia. Habrá que tomar partido en este caso. Apoyado en la teoría social de los campos de Bourdieu (2000), y en atención a Rozengardt (s.f.), y Reyes (2016a), se aborda la Educación Física como un campo social, considerando que la disciplina sugiere linealidad, hermetismo, es ordenadora, además de estructurada y estructurante; la ciencia produce conocimientos, mientras que la Educación Física está mucho más asociada con una intervención educativa que se sirve de las ciencias (así, en plural). En este sentido, la noción de campo a la cual se tributa hace alusión a una dimensión de la realidad asociada a cierto tipo de valores, formas de proceder, prácticas, creencias, representaciones, imaginarios, saberes, lenguajes, actitudes, problemas, teorías, principios, entre otros elementos, que, como sostiene Bourdieu (2000), se articula con otros campos o dimensiones de la realidad.

Se parte del supuesto que sugiere (en autores que ya comentaremos) un agotamiento epistémico de la Educación Física y señala el emerger de la motricidad humana como paradigma científico, dando cuenta de aquello que la Educación Física 'probablemente' ha 


\section{MOTRICIDAD HUMANA: ALGO MÁS QUE UN GIRO LINGÜÍSTICO \\ ¿Qué, del cuerpo y la Educación Física?}

Alixon David Reyes Rodríguez

pp. 54-84

obviado en el terreno de las prácticas que se le adjudican históricamente (más allá de lo planteado discursivamente): esto es, la atención desde la concepción de la integralidad y la complejidad humana. La asunción de la categoría 'cuerpo', en asociación con la de 'ser humano' es un tema central en este debate, y la perspectiva no es reducirle a objeto, a materia orgánica subordinada y subordinable, o a sustituto de la conciencia (Ramírez, 2017), sino pensarlo desde la perspectiva de la complejidad (Da Conceição, 2008). Es así en tanto se trata de un concepto que se viene reconfigurando con el tiempo a merced de las tendencias y corrientes de pensamiento, y como lo sostiene Le Breton (2002:27): "El cuerpo parece ser algo evidente, pero, finalmente no hay nada menos difícil de penetrar que él”.

Para comenzar, necesariamente debe reconocerse que, en este campo, esto es, la Educación Física (utilizando la metáfora espacial de 'campo' empleada por autores como Bourdieu, 2002, y Lewin, 1946), existe una especie de desapego y desinterés por la reflexión epistémica y conceptual (Peña, 2018; Quiroz, 2017; Reyes, 2016a; Rey\&Canales, 2007). Probablemente esto viene dado por el escaso énfasis en procesos de lectura, por la hiperespecialización, por la misma lógica que se instaura a causa de las necesidades del mercado, por la escasa vinculación que de estos saberes hacen los profesores (programas y diseños) con otros campos del saber que podrían ayudar a ampliar las formas de aproximación y comprensión, y por la tendencia a impulsar una Educación Física mucho más orientada por el higienismo, que una Educación Física pensada desde el contexto sociocultural. De allí que no se trate entonces de un debate minúsculo, por el contrario, estamos ante la consolidación de propuestas epistémicas y praxiológicas que potencian lo que se gesta en el contexto de la educación.

\section{CENTRO DE INTERÉS}




\section{MOTRICIDAD HUMANA: ALGO MÁS QUE UN GIRO LINGÜÍSTICO ¿Qué, del cuerpo y la Educación Física?}

Alixon David Reyes Rodríguez

pp. 54-84

¿De qué se habla cuando se trata de la motricidad humana?, ¿por qué se inmiscuye este término en el campo social de la educación? Pues, aunque el pivote de discusión en este paper sea el de la motricidad humana como posibilidad paradigmática y científica, es importante destacar que estamos partiendo de una coordenada que ha sido bautizada y estandarizada históricamente como 'Educación Física'. Y estos campos o dimensiones de formación vienen contextualizándose a su vez en dos formas de asunción o concepciones que se tienen del ser humano, de la educación y del conocimiento a través de la historia; son dos visiones de mundo, de la realidad y del ser humano.

Ahora bien, ese es el punto de inicio básicamente por dos cosas: 1) porque ha sido a la Educación Física a la que se le ha encargado [partiendo de los siglos XVI-XVII según Reyes, A; Reyes, C; Reyes, A. C., 2016 (en lo sucesivo Reyes, et al.), Reyes, 2012; o de los siglos XVII-XVIII según Sérgio, 2006, y Tefarikis, 2006]'todo' lo relacionado con el desarrollo de 'lo corporal' (que en el sentido de lo que ha de desarrollarse se comprenderá que se trata de una exageración de características no menores); y,2) porque la Educación Física es reconocida como 'área de formación' (Ministerio del Poder Popular para la Educación, 2015) y como dimensión curricular en la gran mayoría de los países del mundo, atendiendo a que, como sostiene D’Amico, et al.(2014), hay países en Europa en los que a esta dimensión formativa se le denomina 'Deporte Escolar'.

A la pregunta: ¿por qué se considera una exageración endilgar el desarrollo de 'todo lo corporal' a la Educación Física?, responderíamos que, la Educación Física no puede abarcar ni agotar la totalidad de la cultura humana (Reyes, 2012).En segundo término, porque tal hipótesis indica que a 'las otras educaciones' (término homologado desde la escuela moderna que supone la existencia de áreas, modalidades, entre otros, generando cierta idea de fragmentación) no les corresponde la enseñanza y el desarrollo de experiencias corporales, como que si alguna experiencia humana no fuese corporal. Así las cosas, se genera una diatriba: integralidad versus fragmentación. La cosmovisión de 


\section{MOTRICIDAD HUMANA: ALGO MÁS QUE UN GIRO LINGÜÍSTICO ¿Qué, del cuerpo y la Educación Física?}

Alixon David Reyes Rodríguez

pp. 54-84

realidad, del mundo, de la idea de conocimiento, del ser humano, de la educación, de cuerpo, vienen teñidas por alguna de estas formas de asumir la vida. Ahí se encuentra parte de la relación opuesta que enfrenta a las perspectivas y miradas de la Educación Física y las denominadas ciencias de la motricidad humana. Y ello considerando que, al hablar de integralidad hablamos de una visión de totalidad desde lo complejo, de asunción de una generalidad que reconoce la unidad en medio de la diversidad, de un conjunto complejo pero armónico al tiempo que dialéctico. No obstante, pensar en la fragmentación nos conduce justo a eso, a fragmentos, a partes, a fronteras, límites, compartimentaciones.

\section{Cultura e influencia}

Historiadores reconocen en civilizaciones pasadas (China, Egipto, Mesopotamia, Medopersia, Grecia, Roma) el desarrollo de manifestaciones y expresiones movimentales con intencionalidades diversas como propuestas epocales con sus consabidas diferencias culturales. Entre algunos de los que apuntan a ello están: García (2015), Ruíz, Chincilla y López (2010), Ramírez (2009), Betancor y Vilanou (1995), Guillet (1971), Diem (1966), etc. Junto con Ramírez (2009), se percibe un punto destacable con respecto a quienes incorporan a estos análisis y recuentos históricos algunas otras sociedades y enfoques que generalmente pasan desapercibidos.

Nos referimos a sociedades de pueblos originarios, africanas, asiáticas, esquimales (entre otras), es decir, sociedades consideradas primitivas y periféricas; además de otros enfoques, esto es, la incorporación de temas como las formas de acumulación, los modos de producción, el tema del esclavismo, el trabajo, la revolución francesa, la revolución industrial, la irrupción del mercantilismo y el capitalismo en asociación con el deporte y la actividad física, etc. De allí que se destaquen autores como Hernández y Jova (2013), Oteros (2006), Blanchard (1995), Blanchard y Cheska (1986), Engels (1976), Lukas (1973), Reclus (1905), etc.

Hay quienes se animan incluso a adjudicar a estas culturas el origen de la Educación 


\section{MOTRICIDAD HUMANA: ALGO MÁS QUE UN GIRO LINGÜÍSTICO \\ ¿Qué, del cuerpo y la Educación Física?}

Alixon David Reyes Rodríguez

pp. $54-84$

Física, tal y como puede destacarse en Bastidas, Arteaga y Ascanio (2016), Díaz (2013), Navarro (2010), Salas (1984), Castro (s.f.), entre otros. Pero, vale destacar que en realidad no podríamos hablar de Educación Física propiamente dicha para las épocas en las que las culturas ya comentadas se desarrollaban (Reyes, 2012; Toro, 2007; Tefarikis, 2006) en tanto como destaca Rozengardt (2006), "la intervención sobre esas prácticas con un sentido educativo cobra sentido en los momentos de desarrollo de las culturas urbanas y la gran presencia del Estado, sistematizándose y definiéndose en la modernidad, con un gran impulso en el siglo XIX" (p. 159). El tema es que no se trata solo del surgimiento terminológico y semántico de la Educación Física [que data de los siglos XVI y XVII (Reyes, 2012)], sino de la asunción de la proyección y las intencionalidades pedagógicas o no que estas manifestaciones tuviesen.

En culturas tan definitorias para la humanidad como China, Egipto, Mesopotamia, Medopersia, Grecia, Roma, pueblos originarios, poblaciones africanas, asiáticas, esquimales, etc., existían sistemas de adiestramiento en los que se evidenciaban manifestaciones atléticas y gimnásticas, manifestaciones asociadas a diversas variantes dela lucha y similares, manifestaciones que tenían entre sus propósitos varios la preparación física para el futuro militar, la supervivencia, la adoración politeísta, juegos y otras actividades lúdicas, competencias con fines nupciales, manifestaciones rituales y orgiásticas dionísiacas, danzas, festividades y la celebración de competencias específicas. Por ejemplo, encontramos en Grecia elementos como el areté, la kalokagathia, la paideia, el tema de la virtud, la moral, la verdad, la ética estética, etc. No obstante, consideramos que su invocación no es suficiente como para consolidar la sinopsis esencial de un ideario (como en el caso que nos atañe: la Educación Física) que preló durante siglos y que ha permeado la historia hasta nuestro presente.

Necesario es cotejar elementos de distinción entre las culturas: por ejemplo, en la antigua China se asume una concepción de ser humano desde la representación de la metempsicosis (muy similar al posterior pensamiento órfico asociado a la idea de la 


\section{MOTRICIDAD HUMANA: ALGO MÁS QUE UN GIRO LINGÜÍSTICO ¿Qué, del cuerpo y la Educación Física?}

Alixon David Reyes Rodríguez

pp. $54-84$

reencarnación). Algo similar ocurre en Egipto, cultura politeísta en la que el alma parte a un mundo superior o inferior en relación con la honra de los dioses y el comportamiento humano. Quizá entre todas estas culturas, la mesopotámica, aunque importante y potente, fue la que menos ha impactado en la actualidad al ser comparada con la influencia de aquellas otras como la griega o la romana, incluyendo la cultura judeocristiana. Pero por supuesto, todas estas en su momento de mayor esplendor han ejercido alguna influencia en el mundo. Sin embargo, ninguna de estas llegó a tener un alcance y un impacto tan profundo en la historia de la humanidad como la griega, seguida muy de cerca por la cultura romana (Rodríguez, 2013; González, 2010).

Desde la idea de democracia, la cultura griega ha implicado la asunción de formas de asumir la realidad y el mundo de manera bastante particular. Grecia es considerada como la cuna del dualismo antropológico, base conceptual y filosófica para comprender todo este tema de la irrupción de la Educación Física en el escenario pedagógico y posteriormente de la motricidad humana como posibilidad en el contexto pedagógico y científico.

Hablar de dualismo antropológico implica una concepción del mundo que considera al 'hombre' como centro del universo, a un 'hombre' visto desde una perspectiva androcéntrica, como complemento de una compartimentación, esto es, en Sócrates, Platón, Aristóteles, Plotino, surgen como puntales del ideario griego (siendo que para Platón, el cuerpo era la cárcel del alma, y en Homero el 'soma', o cuerpo, es el cadáver), y planteaban una escisión del ser humano (cuerpo, alma, espíritu) que ha tenido un impacto intergeneracional. Tal impacto se nota hasta en la mismísima aparición de religiones varias. Cada religión tiene una concepción definida con respecto al ser humano, y esa misma concepción convertida en doctrina se vierte sobre el imaginario social y cultural de miles y hasta millones de fieles y devotos en todo el mundo. Así que, es de comprender que las concepciones, las creencias y las representaciones sociales no son neutras, esto es, hay un ejercicio biopolítico que demarca la concepción de la vida y las formas de vivir, y el cuerpo ha sido mediatizado en toda esta esfera de discusión. 


\section{MOTRICIDAD HUMANA: ALGO MÁS QUE UN GIRO LINGÜÍSTICO ¿Qué, del cuerpo y la Educación Física?}

Alixon David Reyes Rodríguez

pp. $54-84$

Vale destacar que el cristianismo tuvo que afrontar decisiones importantes. Por ejemplo: el cristianismo predicado por los apóstoles de Jesucristo fue incisivo en denunciar entre sus seguidores la influencia marcada de la cultura politeísta griega. De acuerdo con Baschet (1999:04):

Hay que recordar que la representación dual de la persona humana -alma y cuerpo- no es un invento del cristianismo. Esta representación ya es la del platonicismo, que pesa muy fuertemente en la teología cristiana, y al cual se le puede calificar de dualista.

El cristianismo apostólico fue permeado por una iglesia que se constituyó de esos esfuerzos primarios de evangelización. Esa iglesia consiente con Roma (poder político imperante), se convierte en su bastión y desarrolla en Europa una creciente concepción del ser humano y del cuerpo que se casa con las mismas alocuciones grecorromanas (Harmon de White, 1999). La iglesia y sus representantes comienzan a asumir posturas encontradas, tal es el caso de San Agustín, quien tuvo que superar en sí mismo dos concepciones en el tiempo, primero al sostener que el alma es un alma racional y que se sirve del cuerpo, y luego pasando a sostener que el alma es una tríada que intenta él explicar desde los registros de los escritos del apóstol Pablo en sus cartas a los cristianos en Corinto. Santo Tomás asume que alma y espíritu son lo mismo (Weber, 1991), mientras que en otros estamentos de la iglesia no sucede así. Surgen referencias a un infierno, a un purgatorio, a un limbo, que no existían en la historia judía ni en el primigenio asentamiento de la iglesia apostólica, implicando esto que la evolución del pensamiento cristiano fue permeado, secularizado y mediatizado por la influencia de las culturas griega y romana.

Pasado el tiempo y pasados también Calvino, Wesley, Gerónimo, Lutero (reformadores de la iglesia y posteriormente perseguidos por la misma), en René Descartes se fortalece una idea dispar en el contexto de un nuevo 'ni tan nuevo' dualismo: rescogitans (alma, lo pensante), resextensa (cuerpo, lo material), o sea un dualismo cuerpo-mente, en lo que se ha denominado posteriormente como dualismo ontológico o lógica cartesiana, pero 


\section{MOTRICIDAD HUMANA: ALGO MÁS QUE UN GIRO LINGÜÍSTICO ¿Qué, del cuerpo y la Educación Física?}

Alixon David Reyes Rodríguez

pp. 54-84

que en esencia sigue partiendo de la misma idea de la cultura grecorromana: la fragmentación. Incluso, escribe Descartes (1981:123):

Me consideré en primer término como teniendo un rostro, manos, brazos, y toda esta máquina compuesta de huesos y carne, tal como aparece en un cadáver y a la que designé con el nombre de cuerpo. Además de esto consideré que me alimentaba, que caminaba, que sentía y que pensaba y relacioné todas estas acciones con el alma.

Luego tenemos la era de los anatomistas del siglo XVI, quienes diseccionan el cuerpo humano para su estudio obteniendo una visión un poco más completa de la versión que del cuerpo se ha tenido en la historia (Pedraza, 2010). Llega la era del maquinismo, la revolución industrial, del ejercicio de estructuración de una lógica comercial en la que el cuerpo es asumido bajo la fórmula del cuerpo-máquina que de alguna manera debe asociarse con los máximos niveles de producción. Ese cuerpo-máquina se transmuta como objeto en el ideario de un cuerpo-mercancía y coincide perfectamente con los conceptos marxistas que plantean temas como el valor de cambio y el valor de uso.

Otros tantos como Malebranche, Bacon, Spinoza, Leibniz avanzarían en la misma lógica ontopositivista. En Nietzsche, por ejemplo, el alma se subordina al cuerpo. Y es que no se trata de un dato menor el entender que ambas formas de apreciación (dualismo antropológico y dualismo ontológico) encuentran en una razón fragmentada su motivo de ser. Y, ¿por qué no es un dato menor?: pues, porque esto que viene del mundo de la filosofía tiene relación directa con la educación. La educación es un derecho humano que debe tener la atención del Estado como garante de ese derecho y se sustenta sobre la necesidad de formar a una ciudadanía con las características que se ameritan para dar configuración al Estado que se pretende y, en consecuencia, surge la idea de la educación necesaria y la teoría pedagógica que ha de asumirse.

De acuerdo con Ornelas (2009:83): “Todo sistema educativo está marcado por una doble elección: la de un proyecto de Hombre y de Sociedad". De allí que la concepción que se tenga del ser humano sea tan importante. Si la idea de ser humano que se tiene es una 


\section{MOTRICIDAD HUMANA: ALGO MÁS QUE UN GIRO LINGÜÍSTICO ¿Qué, del cuerpo y la Educación Física?}

Alixon David Reyes Rodríguez

pp. $54-84$

idea fragmentada, pues, la educación no irá a fortalecer otra cosa que no sea idea. Grecia antigua es una fiel expresión de la idea de fragmentación, así lo demuestran las más de dos mil ciudades-Estado o polis griegas existentes después de la liberación de los micénicos y los dorios, destacan dos ciudades: Atenas y Esparta. Gallego (2017) expresa al respecto: "La mayoría de las ciudades griegas tendría un tamaño de 5 a 8 kilómetros de radio y una población de entre 2.000 y 4.000 habitantes, de los que sólo una parte pequeña correspondería a la comunidad de ciudadanos" (p.14). Si consideramos que Grecia terminó configurándose como imperio mundial, comprenderemos que las ciudades-Estado eran muchas y que fueron creciendo y consolidándose a la par que fueron formando alianzas con las grandes ciudades, esto es, Atenas y Esparta, que, independientemente de la guerra posterior con Persia, terminaron yendo a dos guerras fratricidas (Guerras del Peloponeso) en las que los espartanos se impusieron.

Las concepciones que del ser humano se tenían en Atenas y Esparta distaban mucho de ser similares, y más aún, distaban de acercarse a la idea de integralidad que en algún momento algunos autores le han endosado. Así, puede notarse que hay defensores de la tesis que sostiene que en Atenas se pensaba en un equilibrio cuerpo-mente usando el argumento de planteamientos como las invocaciones y prácticas asociadas a la paideia griega, al areté, a la kalokagathia, entre otros elementos que, si bien es cierto tienen una impronta cultural, terminan siendo subordinados, así como creen que el cuerpo se subordina a eso que, siendo intangible, le domina (Jaeger, 2001; Vergara, 1989). La cultura griega es la que potencia con mayor fuerza las pretensiones culturales y ético-políticas del dualismo, con sus respectivos matices entre Atenas, Esparta, Corinto, Tebas, Chipre, Tesalónica, Creta, entre algunas otras polis de importancia. Le Boulch (1977) comenta:

En Grecia, desde el período clásico, apenas las ciencias y letras alcanzaron suficiente desarrollo, vemos despuntar el antagonismo entre la cultura del cuerpo y del espíritu... Lo que existía entre ambos tipos de formación, física y espiritual, no era precisamente una secreta atracción o armonía preestablecida, sino, por el contrario, la más radical hostilidad (p.17). 


\section{MOTRICIDAD HUMANA: ALGO MÁS QUE UN GIRO LINGÜÍSTICO ¿Qué, del cuerpo y la Educación Física?}

Alixon David Reyes Rodríguez

pp. $54-84$

El pensamiento actual que rescata y orienta hacia la aceptación y la asunción del ideario griego como que si este hubiese concebido al ser humano desde la integralidad, es un pensamiento dominado por la lógica racionalista, y esto es característico del positivismo: segregar, fraccionar (Reyes, 2014). Si bien es cierto que en Esparta no hay algo parecido a la idea de integralidad como representación sociocultural en tanto la concepción reinante es una concepción biologicista, la preocupación espartana pasa entonces por la preparación física para el fortalecimiento de la seguridad militar de la polis, comprendiendo un cuerpo-máquina, dando de esta manera una prelación a lo 'físico' (como compartimento del ser), también parece un poco exagerado afirmar que en Atenas se piensa en integralidad. Allí en Atenas existe el gran germen del dualismo.

En Atenas el ser humano es una composición, una yuxtaposición entre dos cosas que marchan en algunos casos en direcciones contrarias. En Platón, el alma (inmaterial y eterna) habita el cuerpo (material y finito). Así lo hereda de Sócrates y de los filósofos órficos, a quienes se les atribuye la idea de la reencarnación o metempsicosis justificando así la inmortalidad del alma, la preexistencia de esta y la subyugación del cuerpo (que se descompone en la muerte y desaparece) al alma.

La idea de educación no puede sustraerse de otras dimensiones de la vida como si fuese un rompecabezas. La educación es un proceso transversal a la vida que permea todas sus dimensiones. Entonces, la educación no puede pensarse sin pensar la idea de libertad, la idea de democracia, la economía, la justicia, entre otros ámbitos definitorios de la vida en sociedad. Sutherland (2010:16), sostiene: "El desconocimiento de la totalidad es la clave del asunto” (p. 16), y Bourdieu (2002), usó el término 'campo' como una metáfora espacial para referirse a una dimensión de la realidad (política, económica, social, cultural, etc.), y ello en tanto en Bourdieu no es posible comprender la realidad desde el fraccionamiento.

Como ya se ha afirmado en otros trabajos (Reyes, 2016b), hay no poca literatura que presenta a Grecia como la sociedad de la democracia paradigma en la historia universal, y 


\section{MOTRICIDAD HUMANA: ALGO MÁS QUE UN GIRO LINGÜÍSTICO ¿Qué, del cuerpo y la Educación Física?}

Alixon David Reyes Rodríguez

pp. $54-84$

no solo esto, sino que se sugiere en no pocos ejercicios textuales, la necesidad de regresar a los ideales de educación y ciudadanía en la democracia griega antigua. A la luz de estos planteamientos vale la pena pensar en el carácter geohistórico de la educación. Y ello por cuanto estamos hablando de contextos, espacios, tiempos, sociedades y concepciones diametralmente opuestas aunque existan elementos que puedan ser rescatados de aquellas experiencias. Pero de allí a que pensemos en aquello como el patrón o modelo exclusivo, pues, no parece ser muy acertado. Muy bien lo dice Gaviria (2010):"lo que llamaban democracia los griegos puede no ser lo que nosotros llamamos democracia; lo que ellos llamaban libertad puede ser muy diferente a lo que nosotros llamamos libertad" (p.179).

En segundo lugar, y teniendo en cuenta que la educación establece las bases socioculturales y políticas en la formación y consolidación de una nación, de una sociedad, comprendemos que la educación y la democracia guardan una estrecha relación. Sabemos incluso que en ocasiones (no pocas) la manipulación de la educación ha servido a causas no tan nobles. Así, es importante entender que la idea de educación y el concepto de ciudadanía en Grecia, especialmente en Atenas, no pueden desamarrase del concepto de democracia del país helénico en tiempos de su mayor esplendor. Si alguno se pregunta, ¿por qué en Atenas?, pues, diremos que, "la democracia ateniense quizás fue la más importante y significativa entre las ciudades-estados de Grecia, por su influencia en el mundo político y filosófico de la época y hasta la actualidad" (Castillo, 2010, p. 7).

La idea de la democracia en Atenas viene de la posibilidad de la participación en los asuntos públicos de la república, no obstante, habría que comenzar a comprender que, aunque sí, es cierto, se trataba de un sistema novedoso para la época, se trataba además de una forma de conducta social y política que se presumía iría decantándose hasta hallar una forma mucho más pura de democracia, también es cierto que esa democracia se basó en una idea un tanto extraña de ciudadanía (aunque aceptable para la aristocracia griega). ¿Por qué extraña?, pues, basta con revisar en qué consistía la idea de ciudadanía griega para encontrar sus fracturas. Por ejemplo: ¿era la ciudadanía un derecho de todas las personas en 


\section{MOTRICIDAD HUMANA: ALGO MÁS QUE UN GIRO LINGÜÍSTICO ¿Qué, del cuerpo y la Educación Física?}

Alixon David Reyes Rodríguez

pp. 54-84

Atenas?: No. ¿Eran las mujeres, ciudadanas en Atenas?: no. ¿Eran las niñas y los niños, ciudadanos en Atenas?: No. ¿Quiénes carecían de ciudadanía?: las mujeres, las niñas, los niños, los extranjeros, las mujeres y los hombres esclavizados. A la sazón, Castillo (2010), sostiene que en las asambleas de ciudadanos "solo podían participar los hombres libres (únicos considerados ciudadanos), se excluía a las mujeres, menores de edad, extranjeros y particularmente a los esclavos; éstos, al no acceder a la condición de ciudadanos, no tenían derechos políticos” (p. 5).

Es de cuidado lo que sostiene Castillo: "aun cuando una porción mayoritaria de este pueblo no tuviera acceso a este derecho". Es decir, la mayoría del pueblo carecía de la condición ciudadana, carecía de derechos. Cortina (2001), a su vez, afirma: "El ciudadano ateniense se vincula a los que, como él, son libres e iguales, y se distancia de los que no lo son” (p. 1). En alusión a esa caracterización de ciudadanía en Grecia, es importante saber que, a pesar de que la idea de democracia e incluso de ciudadanía (en el marco de la democracia) tuviese origen en tal nación, es necesario recordar que de esas realidades políticas estaban totalmente excluidas las mujeres, estaban excluidos los niños y también todas las personas esclavizadas. En Grecia, las mujeres eran concebidas "como meros objetos de deseo y de rechazo" (Molina, 2014), además, debían a estar sometidas y encerradas en su gineceo, permitiéndoseles solo una serie de limitadísimas acciones.

Y Perelló (1995), hablando sobre la educación en Grecia, especialmente en Atenas, agrega: "los no libres no reciben educación" (s.n). Vale la pena detenerse un poco: si los no libres no reciben educación, es de considerar entonces que la mayoría de la gente en Atenas no era libre, por tanto, no tenía acceso a lo que podían determinar como ‘educación'. Una educación exclusiva para una muy reducida población. En Atenas, vista la cuestión desde hoy, es latente una ciudadanía exclusiva, excluyente de las grandes mayorías del pueblo, segregacionista, esclavista, impulsadora de la pederastia como práctica pedagógica normal y moralmente aceptada por la sociedad junto a otras polis o ciudades-Estado como Creta, Esparta (Asarta, 2016; Pascual, 2011), que negaba el derecho, y que además no respetaba la 


\section{MOTRICIDAD HUMANA: ALGO MÁS QUE UN GIRO LINGÜÍSTICO \\ ¿Qué, del cuerpo y la Educación Física?}

Alixon David Reyes Rodríguez

pp. $54-84$

igualdad, era la base de una democracia y de una educación que se convirtió en una panacea, en un ejemplo de democracia para el mundo occidental.

Y por más avanzada que fuese la idea de democracia entre las formas de gobierno de la época, no parece ser muy coherente esa idea de educación en Atenas con una idea de integralidad, y menos aún para los tiempos que vivimos. ¿Cómo hablar de integralidad cuando la exclusión es un signo que marca la relacionalidad ateniense?, ¿cómo adjudicar una idea de integralidad a la educación griega cuando las grandes mayorías no eran libres? Justo a eso, es a lo que llama Dietrich (2001), la paradoja de la democracia.

Si se comprende que la educación establece las bases socioculturales y políticas en la formación y consolidación de una nación, de una sociedad, entenderemos que la educación y la democracia guardan una estrecha relación. Así, la educación tendrá el encargo de formar a los ciudadanos para ese tipo de sociedad, para vivir y consolidar esa idea de democracia que se ha acordado en comunidad. En este sentido, si pensamos en la democracia ateniense, tendremos que pensar en una educación formadora de gestos conductuales legitimadores de la idea de democracia y ciudadanía ateniense. No hay integralidad alguna en la idea de educación en Grecia, por más que se pregone, por más que se defienda. Y estos planteamientos, traspasados a su vez al ámbito del discurso presente, nos conducen a cuestionar la sola idea de una integralidad en el ser humano en las representaciones socioculturales griegas.

Todo lo relacionado con 'lo físico' ha sido considerado de segundo nivel, y ello en tanto remite a 'lo corporal 'como materia orgánica subordinada, y no sería realmente tan importante en tanto se subyuga a la mente, al espíritu, amén de que el alma (mente o espíritu) es la que domina al cuerpo y es este su morada, no más (según apreciaciones lógicas racionalistas). Cuando se observa y/o se escucha cómo se minusvalora la Educación Física en comparación con otras dimensiones de formación tan importantes como matemáticas, historia, lenguaje, ciencias naturales, física, química, etc., nos trasladamos al ideario griego entendiendo cómo está consolidada tal forma de pensamiento. Igual sucedió 


\section{MOTRICIDAD HUMANA: ALGO MÁS QUE UN GIRO LINGÜÍSTICO \\ ¿Qué, del cuerpo y la Educación Física?}

Alixon David Reyes Rodríguez

pp. $54-84$

en la Roma imperial.

\section{¿Educación Física Vs. Ciencias de la Motricidad Humana?}

La Educación Física naciente entre los siglos XVI y XVII (Reyes, 2012) hereda estas formas del pensamiento, y más aún, hereda una carga pesada en tanto la idea grecorromana que prelaba por tantos siglos de imperio religioso consideraba el cuerpo como asiento y origen del pecado. Y es que, a decir de Baschet (1999), el cristianismo medieval reconoce dos entidades fundamentales: el alma y el cuerpo, esto es, la herencia de la cultura grecorromana. Tan es así que, después de la dizque conversión de Constantino al cristianismo y con ello la unión de Iglesia y Estado -más allá del césaropapismo (Weber, 1964), de Justiniano I, de la doctrina de las dos espadas, entre otras tendencias-, se habla ya de una nueva fase en el imperio romano, esto es, una roma papal. Esa iglesia católica, apostólica y romana (como se define a sí misma) en conjunción con el Estado, terminó configurando aquello que llamaron 'Santa Inquisición', y la purga del pecado justificó (para ellos) el castigo al cuerpo porque así se dizque purificaba el alma.

Las concepciones que se tienen sobre el cuerpo son parte de las cosmovisiones de una cultura y son asimismo el soporte profundo de sus manifestaciones ideológicas (Araya, 2006; Comeau, 2004; Douglas, 1970). Si bien es cierto que la Educación Física emerge con un sesgo en tanto se piensa desde la atención de la persona desde 'lo físico', más se complica el tema cuando se enreda en los albores del capitalismo. Baschet (1999) lo plantea: hay importantes significaciones sociales subyacentes en las formulaciones de la relación alma/cuerpo, y dichas formulaciones constituyen una de las matrices ideológicas fundamentales de la sociedad medieval occidental.

Entonces, no es inocente lo que se ha venido configurando. Se habla en este contexto de una Educación Física ahistórica, despolitizada, descontextualizada e instrumentalista, que se hace acompañar de lógicas segregacionistas, desiguales y asincrónicas. Es una Educación Físicaque, a decir de Zemelman (1998), se encuentra encajonada dentro de los 


\section{MOTRICIDAD HUMANA: ALGO MÁS QUE UN GIRO LINGÜÍSTICO ¿Qué, del cuerpo y la Educación Física?}

Alixon David Reyes Rodríguez

pp. $54-84$

límites disciplinarios, y al mismo tiempo ha estado subordinada por la lógica utilitaria del citius, altius, fortius olímpico tras una búsqueda incesante y sin límites del rendimiento en el deporte como motor y garantía de la competencia, el espectáculo y la mediática del show. Así, la Educación Física ha sido convertida en un apéndice del deporte y como consecuencia se desdibuja su naturaleza antropopedagógica. Esta tradición coloca las premisas del deporte y el mismísimo rendimiento como su horizonte y como su límite; por tanto, la sustitución de sus propios fines ha generado una deuda social de magnitudes insospechadas (Reyes, 2016a).

La Educación Física, desorientada, en crisis, ha perdido su identidad (Crum, 2012; Valdivieso, 2007; Gracia, 2007; Crisorio, en Scharagrodsky, 1995), y si bien es cierto que ha generado importantes procesos, también habría que reconocer que ha servido como dispositivo para la regulación de prácticas que proveen experiencias de aprendizaje convertibles en utilitarias (Reyes, 2016a), reducidas y reductoras pasando por varias etapas: cuerpo-cárcel (habitáculo), cuerpo-máquina, cuerpo-objeto, o incluso bajo las lógicas del rendimiento, del biologicismo, del higienismo, generando experiencias alejadas de la posibilidad remota de generar contextos de aprendizaje en los que la reflexión se una a la acción, contextos en los que se transforma el sistema de relaciones imperante.

La Educación Física actual cuestiona poco o nada, y se le reduce teleológicamente a la gestación y domesticación del cuerpo como consecuencia de la imposición de sistemas educativos sometidos a la lógica de un mercado que requiere un cuerpo-máquina, ágil, fuerte, resistente, saludable (por supuesto), pero sumiso y obediente al sistema de control que opera. Es decir, se concretó una Educación Física servil, poco favorecedora para el oficio del pensamiento, mimetizada con la estructura simbólica de un contexto que renace en la ciudad de Atenas en 1896 con todo un ideario dominado por la revolución industrial inglesa. De allí que sea tan previsible la confusión entre Educación Física y deporte. Y acá se aclara que nuestra postura no es aquella postura 'antideporte' ya cuestionada por Torrebadella (2012). 


\section{MOTRICIDAD HUMANA: ALGO MÁS QUE UN GIRO LINGÜÍSTICO ¿Qué, del cuerpo y la Educación Física?}

Alixon David Reyes Rodríguez

pp. $54-84$

A la fecha, la brecha pedagógica se ha ampliado, y no son pocos quienes asumen y denuncian el hecho de que, la Educación Física actual tiene una influencia muy poderosa de los paradigmas mecanicista, fisicalista, positivista y conductista (Angarita, 2014). Por supuesto, no es este un problema exclusivo de la Educación Física, sino que es un problema de la sociedad, de la educación como concepto general. Así las cosas, la Educación Física necesita resignificarse, sí o sí, a menos que espere solo el decreto de su defunción. Larry Locke (escribiendo por allá en 1992), citado a su vez por Crum (2012), sostiene: "Si la EF va a tener una presencia significativa en las escuelas secundarias del siglo XXI, será mejor desechar el modelo dominante...y comenzar de cero" (p.62).

Lo que se pretende es considerar los signos de fractura de una concepción pedagógica y humanista ante los que surgen propuestas y alternativas desde la episteme. Ante esto, Pierre Parlebas ya planteaba la praxeología motriz como 'ciencia' destinada a atender la acción motriz (Parlebas, 2001), y Rivera (2018) sostiene:

Proponemos una visión de educación física a partir del establecimiento de la Motricidad como un conjunto de saberes científicos-humanistas organizados para investigar las acciones motrices (cuerpo, movimiento y pensamiento crítico). El estudio de la Motricidad permite construir un sistema de conocimientos científicos de las actividades motrices y su contribución al desarrollo humano (larga vida saludable, conocimientos, nivel de vida digno). Los campos disciplinares de la Motricidad son la educación física, la recreación, el deporte y las prácticas motrices alternativas (p. 8).

Comprendiendo estos elementos, Sérgio (citado por Trigo, 2015:87), en referencia a la Educación Física y ante lo que propone como 'Ciencias de la Motricidad Humana', sostiene:

La ciencia de la Motricidad Humana $(\mathrm{CMH})$ ha de transformarse en un "ideal de emancipación", ya que ella nace como una lucha contra todos los dualismos, hombre-mujer, señor-siervo, cuerpo-alma, blanco-negro, etc. Nuestra teoría es la más politizada que yo conozco, en el área de las ciencias humanas, porque desenmascara una ciencia que fundamentaba el colonialismo... hacer de la $\mathrm{CMH}$ una llamada a la democratización de los saberes, en un diálogo donde entrarán aquellos a quien le fue robada la voz; y 


\section{MOTRICIDAD HUMANA: ALGO MÁS QUE UN GIRO LINGÜÍSTICO ¿Qué, del cuerpo y la Educación Física?}

Alixon David Reyes Rodríguez

pp. 54-84

una llamada a una epistemología sin marginados, subalternos o excluidos.

Interesante, es que, la perspectiva desde la que aborda el planteamiento de las 'Ciencias de la Motricidad Humana' reside en el acto político de lo educativo y el hecho democratizante del acto pedagógico. Y así continúa:

A la concepción hegemónica que la Educación Física ha ejercido, en todo el mundo, transportada por las relaciones de producción capitalistas, anteponemos nosotros la $\mathrm{CMH}$ que se dice "ciencia" porque sólo puede ser ciencia lo que es libre y libertador. Nuestra práctica de producción del conocimiento se confunde con la lucha de todos los explotados y marginados por un mundo mejor, es decir, ella es anti-capitalista, anti-sexista, anticolonialista (Ídem).

¿Qué de la Educación Física en este contexto?, ¿cómo surge entonces la motricidad humana como posibilidad científica?, ¿suplanta la una a la otra?, ¿se trata de una moda más?, ¿coexisten?, ¿se trata de lógicas binarias? Importante es destacar que en el transcurso de la historia se destaca el uso creciente de expresiones que intentan dar cuenta del objeto de estudio y del rango de acción de la disciplina. Se habla de expresiones tales como: cultura física, cultura corporal, expresión corporal, cultura del movimiento, educación corporal, ciencias del deporte, educación deportiva, pedagogía del deporte, ciencias de los ejercicios físicos y corporales, gimnología, fisiopedagogía, educación psicocinética, antropocinética, educación por el movimiento, educación motriz, psicomotricidad, pedagogía de la actividad física y el deporte, educación física y deportiva, kinantropopedagogía, ciencias de la motricidad humana, ciencias del movimiento humano, praxiología, entre otros (Cecchini, 1993), al fin, términos que son usados para definir un campo del saber que ha sido conocido como educación física desde mediados del siglo XVII, muy a pesar e indistintamente de que algunas de estas concepciones sean abarcantes o insuficientes (Reyes, et al., 2016).

Zamora (2009), plantea que esta situación en realidad no sería tan novedosa, y que el desafío por las propuestas de diversidad terminológica, ha llevado en cierta manera a 


\section{MOTRICIDAD HUMANA: ALGO MÁS QUE UN GIRO LINGÜÍSTICO \\ ¿Qué, del cuerpo y la Educación Física?}

Alixon David Reyes Rodríguez

pp. $54-84$

generar mayor ambigüedad en la significación de sus conceptos. El mismo autor sostiene que la misma denominación "educación física", es motivo de debate actual, en la que algunos propugnan su sustitución, porque el problema no es solo semántico sino praxiológico, epistémico. ¿Quiere decir esto que las ciencias de la motricidad humana se suman al babel terminológico existente? ¿Es tan importante para el profesional de hoy, conocer, comprender e incorporar a su praxis pedagógica, otro concepto para describir lo que en realidad hacemos en el campo?

\section{APROXIMACIONES TENTATIVAS}

Ante toda esta discusión, vale decir que la motricidad humana es mucho más que un giro lingüístico. Y sí, al afirmar esto se asume que el tema se encuentra atravesado por las tramas del lenguaje y por las tramas del poder, en tanto consecuencia y en tanto origen; y es así por cuanto el lenguaje termina somatizándose a partir de la construcción de significados y significantes, y esos significantes configuran a su vez concepciones, creencias, formas de asunción del mundo y la realidad. Entonces, cuando decimos 'educación', ya hay allí una idea preinstalada de lo que se piensa es educación, cuando decimos 'cuerpo', pues, sucede igual. Como sostiene Lanz (2005), las palabras no son neutras.

Las palabras se enuncian desde ciertas plataformas, y esas palabras dicen cosas y esas cosas construyen imaginarios que a posteriori se convierten en realidades que se concretan. A eso le llamó en su momento Robert Kjellén, la biopolítica, o lo que es lo mismo, cómo se organiza la vida del otro desde el constructo de dispositivos de lo político. Foucault (1977b) vendría posteriormente a plantearlo como el ejercicio del control de la vida colectiva desde el espectro de los dispositivos y los usos de la política. Y el lenguaje es una forma introductoria del ejercicio de la biopolítica.

McLaren (1997), en referencia al ejercicio biopolítico que se ejerce sobre el cuerpo, lo plantea como (en plural): "lugares de enunciación y de inscripción cultural, el terreno de 


\section{MOTRICIDAD HUMANA: ALGO MÁS QUE UN GIRO LINGÜÍSTICO ¿Qué, del cuerpo y la Educación Física?}

Alixon David Reyes Rodríguez

pp. $54-84$

la carne donde se inscribe, construye y reconstruye el significado, son el resultado de tradiciones intelectuales y de las formas en como dichas tradiciones nos han disciplinado para entenderlas” (p. 84). Foucault (1993), agrega: “(...) fue en lo biológico, en lo somático, en lo corporal que, antes de todo, invirtió la sociedad capitalista. El cuerpo es una realidad biopolítica" (p. 80). Y Brohm (1982) lo reafirma al plantear la condición política del cuerpo, cosificado y 'capitalizado' por la lógica mercantil desde el contexto del deporte.

Si bien es cierto la motricidad humana es rango de acción de la Educación Física, esta propuesta de Manuel Sérgio, surge en el entrevero de esa babel terminológica y un agotamiento visible y denunciado de la Educación Física como disciplina pedagógica, pero no lo hace solo como un giro lingüístico, aunque al mismo tiempo lo produce. Produce el giro lingüístico en tanto avanza en la construcción de nuevos significantes en el campo de la atención humana, esto es, por cuanto piensa y asume la integralidad, asume el cuerpo como el ser humano y no como una parte componente. Esto implica que se entiende desde las 'ciencias de la motricidad humana' que el ser humano no posee un cuerpo, no tiene un cuerpo que habita, sino que, al contrario, lo es (Nancy, 2003).

Asumir la motricidad humana como posibilidad paradigmática implica comenzar a pensar en la persona como un ser complejo, y al ser complejo, compréndase que viene planteado en infinitivo, es un "ser", o sea, no está hecho, no está listo y terminado, está haciéndose de manera permanente. Esto tiene que ver no solo con el desarrollo de 'lo corpóreo', sino que está asentado sobre la necesidad de desarrollo de lo social, de lo espiritual, de lo psíquico, en fin, de toda la multidimensionalidad humana. Hablamos del carácter, de los sentimientos, de los valores, de las relaciones, de las creencias, etc.

Merleau-Ponty (1974), plantea el cuerpo desde la noción de Husserl, esto es, como el sujeto (Leib, o cuerpo vivido) trascendiendo al cuerpo-objeto (Körper, cuerpo-materia), de lo corpóreo (lo biológico, porque lo es) hacia el ser-carne, el ser-conciencia, el serpensamiento, el ser de la subjetividad. Siendo así, el acto pedagógico no es un acto conclusivo en asunción de un cuerpo-materia, porque es sujeto. Debe ser un acto que ayude 


\section{MOTRICIDAD HUMANA: ALGO MÁS QUE UN GIRO LINGÜÍSTICO ¿Qué, del cuerpo y la Educación Física?}

Alixon David Reyes Rodríguez

pp. $54-84$

a encontrar siempre líneas de fuga en lo dado como predeterminado. Pero no solo se trata de que es un ser que está haciéndose a diario, sino que además es complejo.

Asumir la motricidad humana como realidad epistémica implica entender que todo está interconectado, que el movimiento no es un fin en sí mismo, sino que trasciende para garantizar la consolidación de la condición humana en una esfera pública desde donde se reconfigura en posibilidades colectivas. Esto es, el aprendizaje colaborativo y solidario son punto de partida y horizonte, no como elemento estático sino como uno que fluye. Al estar interconectado, el acto pedagógico nos ayuda a pensar en su desterritorialización, y sí, un poco también en la idea de curriculum como espacio público en el que todo y todos podemos construir.

Asumir la motricidad humana implica entender el acto pedagógico como un acto complejo, democrático y democratizante, como un acto generador de participación, de inclusión real, de equidad y justicia. Implica para despecho de algunos la democratización y la multilateralidad del poder y la voz en el espacio aúlico en tanto se hace conciencia del propio cuerpo en relación con las y los demás, en relación con las posibilidades corporales emergentes clausurando aquellas predeterminadas que el profesor domina con relativa sapiencia, en relación con las posibilidades que en el entorno social y cultural renacen y se configuran, en relación con la transformación del sistema de relaciones imperante en el marco de la cultura escolar, en relación con prácticas sujetadoras del cuerpo en tanto ser, en relación con las posibilidades de constituirse como el 'nosotros' desde la solidaridad y el aprendizaje colaborativo. Es decir, hay diálogo de los cuerpos, hay reflexividad, valores, comportamientos, hay conciencia e identidad.

La motricidad humana no es un capricho disciplinar o 'la' moda, tampoco es una corriente, no es una tendencia, es una realidad orgánica que debe ser atendida y a la que se le debe prestar atención en el campo de la pedagogía. Ahora, ¿es esta una ciencia?: gran parte del mundo académico viene aceptando esta moción, otros le cuestionan, pero lo que sí es seguro es que se constituye como un campo de tensiones y paradojas, así como Toconás 


\section{MOTRICIDAD HUMANA: ALGO MÁS QUE UN GIRO LINGÜÍSTICO \\ ¿Qué, del cuerpo y la Educación Física?}

Alixon David Reyes Rodríguez

pp. 54-84

(2010) sostiene sea el campo de la Educación Física (campo de luchas) desde su abordaje epistémico.

Manuel Sérgio, quien propone la motricidad humana como ciencia, se apoya en las tres grandes categorías de ciencias que caracteriza el lógico y filósofo belga Jean Ladrière, esto es, las ciencias formales, las ciencias empírico-formales o de la naturaleza y las ciencias hermenéuticas o humanas. Y Sérgio apunta (2006): "Una epistemología de la Motricidad Humana no puede olvidar que no es un área de físicos, sino de personas en movimiento intencional de trascendencia (o superación). Aquí, lo físico está integrado, pero superado" (p. 20).

.De esta forma, la propuesta de la motricidad humana se genera desde el diálogo interdisciplinar como necesidad para la comprensión y abordaje de la complejidad humana. Esto trae como punto de honor el debate que considera un agotamiento progresivo de la Educación Física. Y probablemente tenga aún que constatarse un achique en el rango de acción disciplinar de la Educación Física, y ello en cuanto es de reconocer que en algún punto se ha mostrado reticente al diálogo interdisciplinar, y ello le ha empobrecido (Reyes, 2016a). Más allá de que la declaración de objetivos de la Educación Física sea hermosa, esta se ha acorazado mostrándose hermética a la posibilidad dialógica y constituyendo su mundo en el contexto de la cancha, el laboratorio, el metro y el silbato.

Necesitamos preguntarnos con mayor audacia cuánto sentido tiene seguir hablando de Educación Física como lo hacemos hoy, si alguna otra concepción podrá potenciar la superación de dicotomías y lógicas binarias que hoy entorpecen y limitan la comprensión de lo humano. Y como pregunta Pinillos (2007): “¿Quedará la Educación Física subsumida por esta visionaria propuesta?, ¿se trata de una nueva especie de ciencia madre?, o más bien ¿se intenta realizar un desplazamiento del concepto de Educación Física, a partir de un enfoque omnicomprensivo...?” (p. 20).Almonacid (2012), a la sazón, sostiene:

(...) la Motricidad Humana sin Educación Física no tendría historia, vale decir, la MH tiene su génesis en lo que conocemos desde hace 300 años como 


\section{MOTRICIDAD HUMANA: ALGO MÁS QUE UN GIRO LINGÜÍSTICO ¿Qué, del cuerpo y la Educación Física?}

Alixon David Reyes Rodríguez

pp. $54-84$

Educación Física. La propia Educación Física sin la Motricidad Humana no tiene futuro, puesto que requiere con urgencia una transformación de carácter ontológico, epistemológico y didáctico (pp. 185-186).

Este debate debe permitirnos llegar a una apuesta práxica por la integralidad, la democratización y desterritorialización de los espacios escolares, por la bidireccionalidad en los procesos de enseñanza y aprendizaje, por la resignificación de lo lúdico, por la equidad social en el entendido de la generalidad de la población en todos los contextos, por el diálogo y el abordaje interdisciplinar de lo educativo. Ello implica la descolonización de prácticas corporales, la generación de condiciones para el desarrollo de un pensamiento crítico, para la reflexión permanente en/sobre la práctica pedagógica, para el ejercicio de la justicia en el sistema de relaciones que inicia su constitución y su consolidación por la familia, los pares, la escuela (y de manera primigenia desde la sesión de clase ya no asumida como tal sino como un encuentro de personas para el aprendizaje colectivo y colaborativo), los medios masivos de información, entre otros ámbitos.

La motricidad humana es responsabilidad de todos, incluyendo la Educación Física. No es responsabilidad exclusiva de algún campo. Tiene que ver con una responsabilidad compartida, incluso por la sociedad. De allí que, asumirla como realidad epistémica y científica, nos llevaría a la transformación del campo educativo partiendo de nuevas visiones y abordajes en la práctica profesional, nos llevaríaa militar en el terreno de las tensiones socioculturales, y costaría el destierro de muchas convenciones sociales, culturales, políticas y económicas hoy.

\section{REFERENCIAS}

Almonacid F., A. (2012). La educación física como espacio de transformación social y educativa: perspectivas desde los imaginarios sociales y la ciencia de la motricidad humana. Estudios pedagógicos, XXXVIII, Número Especial 1, pp. 177-190.

Angarita, C. (2014). Comprensión epistémica de la Educación Física desde la comprensión del ser (Educa-Fiser). ARJÉ, Revista de Postgrado FACE-UC, 8, (14), pp. 273-303. 


\section{MOTRICIDAD HUMANA: ALGO MÁS QUE UN GIRO LINGÜÍSTICO \\ ¿Qué, del cuerpo y la Educación Física?}

Alixon David Reyes Rodríguez

pp. $54-84$

Araya E., A. (2006). El castigo físico: el cuerpo como representación de la persona. Un capítulo en la historia dela occidentalización de América, Siglos XVI-XVIII. Historia 39, (2), pp. 349-367.

Asarta, U. I. (2016). La pederastia institucionalizada en la sociedad espartana. Actas del III Congreso Internacional de Jóvenes Investigadores del Mundo Antiguo CIJIMA III, pp. 233-248.

Baschet, J. (1999). Alma y cuerpo en el Occidente medieval: una dualidad dinámica, entre pluralidad y dualismo, en, Baschet, J.; Pitarch, P.; Ruz, M. H.Encuentros de almas y cuerpos, entre Europa medieval y mundo mesoamericano. Universidad Autónoma de Chiapas: México, pp. 41-83.

Bastidas, G.; Arteaga, E.; Ascanio, A. (2016). Educación física y deporte su evolución en períodos históricos mundiales y de Venezuela como base para la teorización de estudios de postgrado en la Universidad de Carabobo. Atrio, 4, (3), pp. 6-12.

Betancor L., M. A.; Vilanou T., C. (1995). Historia de la Educación Física y el deporte a través de los textos. Barcelona: PPU.

Blanchard, K. (1995). Theanthropologyof sport: anintroduction. Westport: ConnPraege.

Blanchard, K.; Cheska, A. (1986).Antropología del deporte. Barcelona: Bellaterra.

Bourdieu, P. (2002). Campo de poder, campo intelectual. Itinerario de un concepto. Argentina: Editorial Montressor.

Castillo, H. (2010). Democracia y salud: la plusvalía psicológica. Caracas: Monte Ávila Editores Latinoamericana.

Castro, M. (s.f.). Educación Física, una mirada desde la filosofía. Revista Motricidad y Persona, 10, pp. 45-50.

Cecchini, E., J. A. (1993). Antropología y epistemología de la Educación Física.Oviedo: Ferrería.

Cortina, A. (2001). Ciudadanía política: del hombre político al hombre legal. Sin mayores datos filiatorios.

Crisorio, R. (2007). Educación Física e identidad: conocimiento, saber y verdad, en, 


\section{MOTRICIDAD HUMANA: ALGO MÁS QUE UN GIRO LINGÜÍSTICO \\ ¿Qué, del cuerpo y la Educación Física?}

\section{Alixon David Reyes Rodríguez}

pp. $54-84$

Chaverra F., B. E. \& Uribe P., I. D. (Eds.). Aproximaciones epistemológicas y pedagógicas a la Educación Física. Un campo en construcción.Colombia: Funámbulos Editores.

Crum, B. (2012). La crisis de identidad de la Educación Física: Diagnóstico y explicación. Educación Física y Ciencia, 14, pp. 61-72.

D’Amico, R. L. de; Yan Ho, W. K.; Branislav, A.; Dinold, M.; Benn, T.; Holzweg, M. (2014). Voces acerca de la Educación Física en América Latina. Revista Desencuentros, Vol. II, pp. 6-14.

Descartes, R. (1981). Meditaciones metafísicas.Madrid: Espasa Calpe.

Díaz, H. L. (2013). Visión sinóptica de la historia de la Educación Física. Revista Motricidad y persona, 12, pp. 41-45.

Diem, C. (1966). Historia de los deportes. Volumen I. Barcelona: Luis de Caralt.

Dietrich, H. (2001). Bases de la democracia participativa. Portuguesa: Alcaldía de Páez.

Douglas, M. (1970). Símbolos naturales. Exploraciones en Cosmología. Madrid: Editorial Alianza.

Engels, F. (1976). El origen de la familia, la propiedad privada y el Estado. La Habana: Editora Política.

Foucault, M. (2013). El orden del discurso. México: Tusquets.

Foucault, M. (2002). La arqueología del saber. Buenos Aires: Siglo XXI Editores.

Foucault, M. (1977a). Microfisica do poder. Río de Janeiro: Graal.

Foucault, M. (1977b). Historia de la sexualidad, Vol. 1. Buenos Aires: Siglo XXI Editores.

Foucault, M. (1976). Vigilar y castigar. Buenos Aires: Siglo XXI Editores.

Gallego, J. (2017). La pólis griega: orígenes, estructuras, enfoques. Buenos Aires: Editorial de la Universidad de Buenos Aires.

García R., F. (2015). Deporte y educación en la Grecia Clásica. Materiales para la historia 


\section{MOTRICIDAD HUMANA: ALGO MÁS QUE UN GIRO LINGÜÍSTICO \\ ¿Qué, del cuerpo y la Educación Física?}

Alixon David Reyes Rodríguez

pp. $54-84$

del deporte. Suplemento Especial, 2, pp. 17-36.

Gaviria D., C. (2010). La democracia en Grecia clásica y su relación con la democracia moderna. Criterio Jurídico Garantista, 2 (3), pp. 174-187.

Gracia D., A. J. (2007). La crisis silenciosa de la Educación Física: un problema epistemológico-conceptual de la Educación Física. Revista Corporeizando, 1, (1), pp. $1-20$.

Guillet, B. (1971). Historia del deporte. Barcelona: Oikos-Tau.

Harmon de White, E. G. (1999). Conflicto inminente entre Cristo y Satanás. Estados Unidos de América: HarvestimeBooks.

Hernández M., P. L.; Jova E., L. (2013). La actividad física en la comunidad primitiva. Lecturas EFDeportes, Año 18, $\mathrm{N}^{\circ}$ 179. Consultado el 18-09-2018. Disponible en línea: $\quad$ http://www.efdeportes.com/efd179/la-actividad-fisica-en-la-comunidadprimitiva.htm

Jaeger, W. (2001). Paideia: los ideales de la cultura griega. México: Fondo de Cultura Económica.

Le Boulch, J. (1977). La Educación por el Movimiento. Buenos Aires: PAIDOS.

Le Breton, D. (2002). Sociología del cuerpo. Buenos Aires: Ediciones Nueva Visión.

Lewin, K. (1946). Actionresearch and minorityproblems, en, Gertrude W., Lewin (Ed.), Resolving Social Conflict.London: Harper\&Row.

Lukas, G. (1973). Educación Cultural Prehistórica. Citius, Altius, Fortius, pp. 273-324. Madrid: Instituto Nacional de Educación Física y Deporte.

McLaren, P. (1997). Pedagogía crítica y cultura depredadora. Políticas de oposición en la era posmoderna. Barcelona: Editorial Paidós.

Merleau-Ponty, M. (1974). Fenomenología de la percepción. Barcelona: Península.

Ministerio del Poder Popular para la Educación (2015). Proceso de Transformación Curricular en Educación Media. Caracas: MPPE. 


\section{MOTRICIDAD HUMANA: ALGO MÁS QUE UN GIRO LINGÜÍSTICO \\ ¿Qué, del cuerpo y la Educación Física?}

Alixon David Reyes Rodríguez

pp. $54-84$

Molina R., G. (2014). La mujer en Grecia y Roma. Ponencia presentada en el VI Congreso Virtual sobre Historia de las Mujeres, 15 al 31 de octubre de 2014, Jaén, España.

Nancy, J.-L. (2003). Corpus. Madrid: Arena Libros.

Navarro, J. (2010). Proceso histórico de la Educación Física. Caracas: FEDUPEL.

Ornelas D., J. (2009). Neoliberalismo y capitalismo académico, en, Gentili, P. et al. (Comps.). Políticas de privatización, espacio público y educación en América Latina. Argentina: CLACSO \&HomoSapiens Ediciones.

Ostoic, Z. (1983a). Problemática corporal y Educación Física. Educación Física Chile, 176, p. 9.

Oteros, A. (2006). Análisis antropológico del juego en las sociedades primitivas. Revista I+E. Investigación y Educación. III, (26), pp. 1-8.

Parlebas, P. (2001). Juego, deporte y sociedad. Léxico de praxiología motriz. España: Paidotribo.

Pascual V., S. (2011). La pederastia griega y el eros platónico. Avances de Investigación, Junio2011. Uruguay: Facultad de Humanidades y Ciencias de la Educación.

Pedraza, Z. (2010). Del cuerpo. Revista Javeriana, 77 (770), pp. 8-15.

Peña R., E. (2018). "Pensar no es lo mío..." Las actitudes en y hacia los procesos de enseñanza y aprendizaje de estudiantes de primer semestre del programa de Ciencias de la Actividad Física y del Deporte. Tesis de Maestría inédita. Universidad Mayor de San Simón, Bolivia.

Perelló, J. (1995). Apuntes de Historia de la Educación. 2a ed. Quito: UPS.

Pinillos G., J. M. (2007). La pedagogía y la motricidad humana, una condición de posibilidad para la construcción de una episteme de la educación física en la perspectiva del desarrollo humano en Colombia. Lúdica Pedagógica, 2, (12), pp. 1930 .

Quiroz S., A. A. (2017). Hábitos de Lectura en Formadores de Formadores del INSEF. Revista Científica de Publicación CEPIES. 2, (1), pp. 74-78. 


\section{MOTRICIDAD HUMANA: ALGO MÁS QUE UN GIRO LINGÜÍSTICO \\ ¿Qué, del cuerpo y la Educación Física?}

Alixon David Reyes Rodríguez

pp. $54-84$

Ramírez T., J. (2009). Fundamentos teóricos de la recreación, la educación física y el deporte. Maracay: Episteme.

Reclus, E. (1905). L’homee et la Terre. France: LibrairieUniverselle.

Rey, A.; Canales, I. (2007). Discurso epistémico para una ciencia de la motricidad. Cinta de Moebio, 28, pp. 117-140.

Reyes, A. (2016a). Pedagogía crítica y Educación Física. Portal Otras Voces en Educación. Consultado el 26-07-2018. Disponible en línea: http://otrasvoceseneducacion.org/archivos/18126

Reyes, A. (2016b). De la Educación Física y sus posibilidades desde la teoría crítica latinoamericana. Revista Ameerijsha, 1, (1), pp. 6-35.

Reyes, A.; Reyes, C.; Reyes, A. C. (2016). Pensar la Educación Física. Diálogos Pedagógicos, Año XIV, 27, pp. 107-129.

Reyes, A. (2014). Consideraciones básicas sobre la asunción de conocimiento. Revista Praxis \& Saber, 5, (9), pp. 103-126.

Reyes, A. (2011). Filosofía de las religiones. Un ejercicio hermenéutico a través del relato del Rico y Lázaro. Alemania: LAP LAMBERT \& Editorial Académica Española.

Rivera V., A. (2018). Prólogo, en, Rivera V., A. (Comp.). Actualidades en Educación Física y Deporte Escolar en México. México: Ediciones INNED.

Rodríguez N., J.F. (2013). ¿Qué legó Roma a la identidad cultural de Europa?Revista Historia UdeC, 20 (2), pp. 7-42.

Rozengardt, R. (2006). (Comp.). Notas históricas para la identidad de la Educación Física, en, Apuntes históricos para profesores de Educación Física. Buenos Aires: Miño y Dávila Editores.

Rozengardt, R. (s.f.). La Educación Física: ciencia, ética, política y pedagogía. Un aporte histórico a la identidad del campo. Sin mayores datos filiatorios.

Salas, S. (1984). Grecia y su legado olímpico. Educación Física Chile, 201, pp. 8-9.

Scharagrodsky, P. (1995). Entrevista al profesor Ricardo Crisorio. Educación Física y 


\section{MOTRICIDAD HUMANA: ALGO MÁS QUE UN GIRO LINGÜÍSTICO \\ ¿Qué, del cuerpo y la Educación Física?}

Alixon David Reyes Rodríguez

pp. $54-84$

Ciencia, Vol. 1, № 0 , pp. 40-45.

Sérgio, M. (2006). Motricidad humana ¿Cuál es el futuro? Pensamiento educativo, 30, pp. 14-33.

Sérgio, M. (2004). Didáctica de la motricidad, un desafío posible, en, Seminario Educación Física y Organización. Chile: UCSH.

Sérgio, M. (1994). Motricidad Humana. Lisboa: Instituto Piaget.

Tefarikis U., E. (2006). Motricidad humana. Un cambio urgente y necesario. Pensamiento educativo, pp. 94-107.

Toconás, S. G. (2010). Contribuciones para una epistemología de la Educación Física. Diálogos pedagógicos, Año VIII, (15), pp. 85-101.

Toro, S. (2007). Memoria y evolución, pilares de una educación consciente desde la motricidad. Revista Profissao Docente, 7, (16), pp. 19-61.

Torrebadella F., X. (2012). El deporte contra la Educación Física. Un siglo de discusión pedagógica y doctrinal en la educación contemporánea. Movimiento Humano, 4, pp. 73-98.

Trigo, E. (2015). (Coord.). Pensar y transformar: un legado de Manuel Sérgio. Colombia: Instituto Internacional del Saber.

Valdivieso A., F. (2007). En busca de la identidad perdida... Venezuela: Instituto Pedagógico de Maracay.

Vergara, F. A. (1989). La paideia griega. UniversitasPhilosophica, 11-12, pp. 153-168.

Weber, M. (1964). Economía y sociedad. Esbozo de sociología comprensiva. México: Fondo de Cultura Económica.

Weber, E-H. (1991). La personnehumaineauXIIIèsiècle. París: Vrin.

Zamora, J. (2009). Epistemología de la educación física. Guatemala: Dirección General de Educación Física del Ministerio de Educación.

Zemelman, H. (1996). Problemas antropológicos y utópicos del conocimiento. México: El 


\section{MOTRICIDAD HUMANA: ALGO MÁS QUE UN GIRO LINGÜÍSTICO ¿Qué, del cuerpo y la Educación Física?}

\section{Alixon David Reyes Rodríguez}

Colegio de México.

Zemelman, H. (1998). Epistemología y política en el conocimiento socio-histórico, en, Maerk, J.; Cabrolié, M. (Eds.). ¿Existe una epistemología latinoamericana? México: Universidad de Quintana Roo. 